\title{
Morbimortalidad asociada a la disfagia en pacientes ingresados en un hospital general universitario
}

\author{
Morbimortality associated with dysphagy in patients hospitalized in a university \\ hospital
}

\begin{abstract}
Abel González-González $\mathbb{D}^{1}$, Rebeca de la Fuente Cañibano $\mathbb{D}^{1}$, Miriam Blanco Ruíz $\mathbb{D}^{1}$, Belén Fernández de Bobadilla Pascual ${ }^{1}$, Marta Entrenas Valle $\mathbb{D}^{1}$, Celia Montoliú Peco ${ }^{1}$, Isabel Domínguez Osorio $\mathbb{D}^{1}$, Pedro Antonio Muñoz $\mathbb{D}^{1}$

${ }^{1}$ Hospital Universitario de Ciudad Real, Ciudad Real, España
\end{abstract}

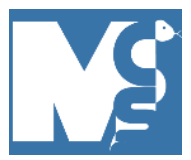

Recibido: $16 / 08 / 2020$

Revisado: 18/08/2020

Aceptado:19/09/2020

\section{Autor correspondiente}

Abel González González Hospital Universitario de Ciudad Real, Ciudad Real - España abelg@sescam.jccm.es

\section{Conflictos de interés}

Los autores declaran no poseer conflictos de interés.

\section{Fuente de financiación}

Los autores no recibieron apoyo financiero para la investigación, autoría y/o publicación de este artículo.

Este artículo es publicado bajo una licencia de Creative Commons Reconocimiento 4.0 Internacional.

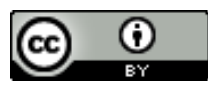

\section{RESUMEN}

Introducción: La disfagia tiene una morbimortalidad importante en pacientes hospitalizados. Objetivos: principal; describir las características de los pacientes con disfagia hospitalizados y, secundarios; cuantificar y analizar la prevalencia de mortalidad y de reingresos. Metodología: Estudio transversal descriptivo de las hospitalizaciones por disfagia durante el año 2015 en un Hospital General Universitario. Resultados: Se evaluaron 431 historias clínicas. La edad de los pacientes fue de 83,21 (DE 11,4) años, el 52,5\% fueron mujeres y el $47,2 \%$ varones; la estancia media fue de 11,1 (DE 7,99) días. En el $71,2 \%$ de los casos la disfagia fue por afectación de la fase orofaríngea. En el $80,51 \%$ de los casos se diagnosticaron complicaciones respiratorias: $48,12 \%$ neumonía aspirativa por líquidos, $40,05 \%$ neumonitis química por aspiración y $11,81 \%$ neumonía aspirativa por sólidos. La mortalidad general asociada a las complicaciones respiratorias respecto del total de los casos de disfagia fue del $24,49 \%$. El $50,48 \%$ de los pacientes con neumonía aspirativa fallecieron. La principal causa de la disfagia fue las enfermedades neurológicas (un 77,25\%). La mortalidad fue significativamente mayor en las mujeres $-42,3 \%$ frente al $7,8 \%-(p<0,01)$ y esta diferencia se mantuvo tras ajustar el resultado por edad: OR 9,937, IC95\%: 5,446; 18,131. El 13,10\% de los pacientes reingresaron al menos en una ocasión. Los pacientes de geriatría presentaron un mayor número de reingresos por número de ingresos. Discusión: las enfermedades neurológicas fueron la principal causa de disfagia. La mortalidad fue significativamente mayor en las mujeres.

Palabras clave: disfagia; disfagia orofaríngea; neumonía aspirativa

\section{ABSTRACT}

Introduction: Dysphagia is an important associated morbidity and mortality in hospitalized patient. Objectives: Main; to describe the characteristics of patients admitted for dysphagia and secondary; quantify and analyze the prevalence of mortality and readmissions. Methodology: Cross-sectional study descriptive revenues by dysphagia during the year 2015 in a University General Hospital. Results: 431 records were evaluated. The age of the patients was 83,21 (11.4), $52.5 \%$ women and $47.2 \%$ male; the average stay was 11.1 (7.99) days. In $71,2 \%$ of cases the dysphagia was involvement of the oropharyngeal phase. $80.51 \%$ of cases were diagnosed respiratory complications. The percentage distribution of these complications were: in $48.12 \%$ aspiration pneumonia due to fluids, in $40.05 \%$ chemical aspiration pneumonitis and in $11.81 \%$ aspiration pneumonia due to solids. The overall mortality associated with respiratory complications compared to the total of cases of dysphagia was $24.49 \% .50 .48 \%$ of patients diagnosed with aspiration pneumonia died. The main cause of dysphagia was neurological diseases $(77.25 \%)$. Mortality was significantly higher in women $-42.3 \%$ of women compared with $7.8 \%$ of males $-(p<0.01)$ and this difference remained after adjusting the result by age: OR $9,937,95 \% \mathrm{Cl}: 5,446 ; 18,131.13 .10 \%$ of patients re-entered at least on one occasion. Patients of geriatric unit that presented in greater number of readmissions by admissions. Discussion: neurological diseases were the main cause of dysphagia. Mortality was significantly higher in women

Keywords: Dysphagia; oropharyngeal dysphagia; aspiration pneumonia 


\section{INTRODUCCIÓN}

La disfagia orofaríngea (DOF) es un síntoma que se refiere a la dificultad de hacer progresar el bolo alimenticio desde la boca hasta el estómago (1); suponiendo una alteración en la eficacia (que predispone a la desnutrición y la deshidratación) y en la seguridad (que puede ocasionar complicaciones respiratorias graves) de la deglución (2).

Se trata de un problema infra-diagnosticado, tanto en hospitales como en otros ámbitos sanitarios y, además, altamente prevalente en la población anciana por su patología concomitante (enfermedades neurológicas y/o neurodegenerativas) y por los cambios que conlleva el proceso del envejecimiento (3).

Su presencia se asocia a un incremento de complicaciones importantes que pueden llevar al paciente a necesitar ingresos hospitalarios frecuentes $\mathrm{y}$, por tanto, a un aumento de la morbimortalidad.

El principal objetivo de este trabajo fue describir las características de los pacientes hospitalizados en nuestro Centro por disfagia: edad, sexo, tipo de disfagia, causas y unidad hospitalaria donde ingresan. Y como objetivos secundarios se plantearon cuantificar la prevalencia de la mortalidad asociada y el del número de reingresos y analizar éstos respecto a distintas variables como la edad, el sexo o la unidad de hospitalización.

\section{METOdOLOGÍA}

Se realizó un estudio transversal descriptivo de las hospitalizaciones por disfagia durante el año 2015 en el Hospital General Universitario de Ciudad Real (España). La revisión de las historias clínicas fue llevada a cabo por todos los autores del trabajo. Se revisaron no sólo los informes clínicos del alta hospitalaria, sino las diferentes anotaciones de los médicos responsables de los pacientes, de los interconsultores e incluso del personal de enfermería, para descartar la ausencia de errores en los diagnósticos codificados. Se desecharon aquellas en las que el diagnóstico no estaba suficientemente documentado. Las variables evaluadas fueron: edad; sexo; tipo de disfagia: oral, oro-faríngea, faringo-esofágica y esofágica; causas de la disfagia: neoplasias de cabeza y cuello (orales, faríngeas, laríngeas y esofágicas), enfermedades neurológicas: vasculares y no vasculares y otras causas; unidad de hospitalización; complicaciones respiratorias e intervención terapéutica nutricional realizada.

Finalmente, también se escogió como variable "reingreso". Se utilizó la definición propuesta por Alonso Martínez el al (4), que considera reingreso (verdadero reingreso o ingreso relacionado) a todo nuevo ingreso hospitalario por el mismo motivo (disfagia o complicaciones derivadas de ésta) durante el periodo evaluado (año 2015). Las variables se han resumido mediante estadísticos descriptivos adecuados a la naturaleza de cada variable: medidas de tendencia central (media) y de dispersión (desviación estándar) para las variables cuantitativas y frecuencias absolutas y relativas (porcentajes) para las cualitativas. La comparación entre las variables se ha llevado a cabo con la prueba Chi cuadrado cuando ambas eran cualitativas y con la prueba t de Student cuando una de ellas era cuantitativa. Como análisis multivariante para controlar las variables confusoras se ha empleado la regresión logística múltiple. El nivel de error alfa o significación estadística escogido fue inferior al $5 \%$. Los cálculos estadísticos se han realizado con el paquete estadístico (PASW 18.0 (SPSS Inc).

El proyecto de investigación titulado "Valoración de la disfagia en pacientes ingresados en el Hospital General Universitario de Ciudad Real" del que se extrajeron los datos expuestos en este trabajo fue aprobado por el Comité Ético de Investigación con Medicamentos de la Gerencia de Atención Integrada de Ciudad Real con fecha del 18 de diciembre de 2018.

\section{RESULTADOS}

Se evaluaron las historias clínicas de 375 pacientes, equivalentes a 431 ingresos hospitalarios. La edad de los pacientes fue de 83,21 (DE 11,4) años, el 52,5\% fueron mujeres y el $47,2 \%$ varones, la estancia media fue de 11,1 (DE 7,99) días y los dos servicios clínicos que acogieron a la mayoría de los ingresos fueron Geriatría (un 69,10\%) y Medicina interna (un 15,1\%).

307 (el 71,2\%) de los casos presentaron disfagia por afectación de la fase orofaríngea. En 21 (el 4,8\%) la afectada fue la fase oral; y en el mismo porcentaje la fase esofágica (4,8\%). En 82 casos (un $19 \%$ ) no se encontró información sobre el tipo de disfagia. En 347 casos (el $80,51 \%$ ) se diagnosticaron complicaciones respiratorias atribuidas a la disfagia (en el momento del ingreso o a lo largo de su estancia hospitalaria). La distribución porcentual de estas complicaciones fue: en el $48,12 \%$ neumonía aspirativa por líquidos, en el $40,05 \%$ neumonitis química por aspiración y en el $11,81 \%$ neumonía aspirativa por sólidos. La mortalidad general asociada a las complicaciones respiratorias respecto del total de los casos de disfagia fue del $24,49 \%$. El 50,48\% de los pacientes diagnosticados de neumonía aspirativa (por líquidos o sólidos) fallecieron.

La principal causa de la disfagia fue las enfermedades neurológicas: 333 casos (un 77,25\%); de los que 210 (un $48,72 \%$ del total de las disfagias) eran de origen 
vascular $123(28,53 \%)$ no vascular. Entre las no vasculares la enfermedad más prevalente fue la enfermedad de Alzheimer y entre las vasculares la demencia vascular (por encima del ictus isquémico). Sólo en 14 de los ingresos (el 3,2\%) la causa fue por un cáncer de cabeza y cuello.

Se realizó una valoración del estado nutricional documentada en sólo en 49 de los casos, de los que 28 fueron diagnosticados de desnutrición mixta, 10 de proteica, 3 de calórica y en el resto no especificaba el tipo. A pesar de esto, en más de 300 casos se realizó algún tipo de intervención nutricional destinada a la modificación de la textura de la dieta, al contenido calórico proteico o al método de administración (Figura 1).

FIGURA 1. TIPOS DE INTERVENCIÓN NUTRICIONAL REALIZADA (N=323)

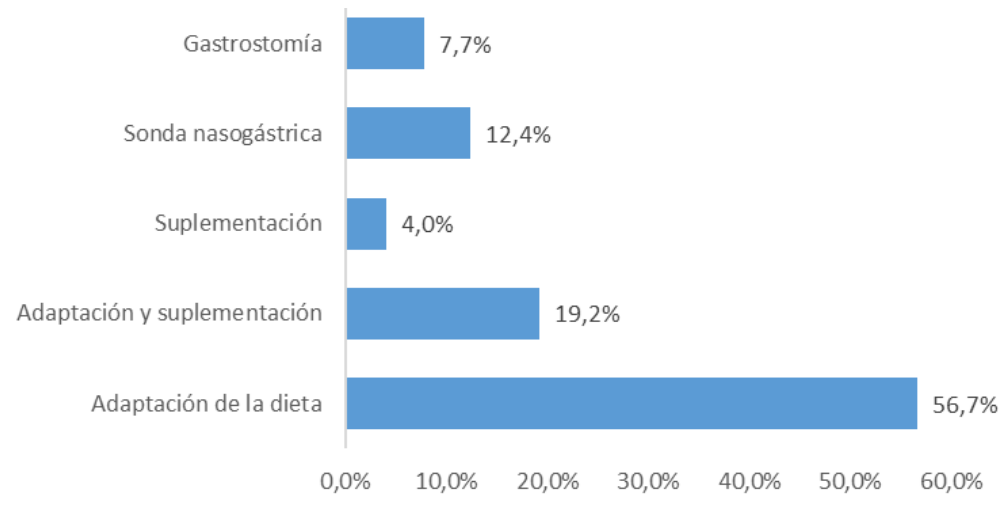

Aunque la edad de las mujeres, 85,35 (DE 10,26) años, fue mayor que la de los hombres, 80,83 (DE 12,13) años, $p<0,001$, no se encontró diferencias en la duración de la estancia hospitalaria entre sexos, 11,13 $(D E 7,15)$ días en hombres frente a 11,07 (DE 8,69) días en mujeres. Sin embargo, la mortalidad sí fue significativamente mayor en las mujeres $-42,3 \%$ de las mujeres frente al $7,8 \%$ de los varones $(p<0,01)$ - y esta diferencia se mantuvo tras ajustar el resultado por edad (regresión logística múltiple): OR 9,937, IC95\%: 5,$446 ; 18,131$.

Los pacientes ingresados en geriatría tuvieron una media de edad mayor que los de otros servicios: 87,96 (DE 5,2) años; en el resto de unidades de hospitalización las medias de edad estuvieron entre 75,8 (DE 11,10) años, los de medicina interna y 58 (DE $39,59)$ años, los de cirugía general y digestiva.

El $13,10 \%$ de los pacientes (49) reingresaron al menos en una ocasión; entre éstos se produjo 1,16 reingresos por paciente. Las diferencias en cuanto a la edad, la duración del primer ingreso y la distribución por sexos entre los pacientes que reingresaron y los que no, quedan recogidos en la Tabla 1.

TABLA 1. DIFERENCIAS DE EDAD Y TIEMPO DE HOSPITALIZACIÓN Y DISTRIBUCIÓN POR SEXO ENTRE LOS PACIENTES QUE REINGRESARON Y LOS QUE NO ( $n=374)$

\begin{tabular}{lccc}
\hline Variables & $\begin{array}{c}\text { Pacientes sin reingresos } \\
(\mathrm{n}=325)\end{array}$ & $\begin{array}{c}\text { Pacientes con reingreso } \\
(\mathrm{n}=49)\end{array}$ & $\mathrm{p}$ valor \\
\hline Edad (años) & $82,36(\mathrm{DE} 12,24)$ & 85,67 (DE 7,97) & 0,014 \\
Días de ingreso & 11,27 (DE 7,88) & 11,57 (DE 7,81) & $\mathrm{p}>0,05$ \\
& & & \\
Sexo (H/M) & $44,61 \% / 55,38 \%$ & $57,1 \% / 42,85 \%$ & $\mathrm{p}>0,05$ \\
& & & \\
\hline
\end{tabular}


En la Tabla 2 se describen las diferencias respecto al número de ingresos hospitalarios, los días de duración de éstos, el número de pacientes reingresados y la mortalidad entre las 5 unidades de hospitalización con mayor número de diagnósticos de disfagia. El 39,76\% de los pacientes evaluados presentaron una mala evolución; considerando ésta el fallecimiento del paciente o el reingreso tras la primera hospitalización.

TABLA 2. DATOS RELATIVOS A LOS INGRESOS HOSPITALARIOS, REINGRESOS Y MORTALIDAD POR DISFAGIA EN LAS 5 UNIDADES DE HOSPITALIZACIÓN CON MAYOR NÚMERO DE DIAGNÓSTICOS.

\begin{tabular}{lcccc}
\hline Unidad de hospitalización & $\begin{array}{c}\text { Ingresos } \\
\text { (número) }\end{array}$ & Días de ingreso & $\begin{array}{c}\text { Pacientes } \\
\text { reingresados }\end{array}$ & Mortalidad \\
\hline Geriatría & 298 & $10,45 \pm 6,92$ & 39 & $31,27 \%$ \\
Medicina interna & 65 & $12,48 \pm 8,51$ & 4 & $47,72 \%$ \\
Oncología médica & 17 & $11,62 \pm 9,87$ & 2 & $6,25 \%$ \\
Unidad de cuidados Paliativos & 15 & $10,33 \pm 5,25$ & 1 & $36,36 \%$ \\
Neurología & 10 & $10,45 \pm 6,92$ & 0 & $25 \%$ \\
\hline
\end{tabular}

\section{DISCUSIÓN}

La disfagia constituye un importante problema en el paciente geriátrico, con gran impacto en la capacidad funcional, en la calidad de vida y en la salud (5). Los pacientes con enfermedades neurodegenerativas, enfermedades cerebrovasculares y los ancianos frágiles son los más susceptibles para padecer disfagia (5) y sus complicaciones $(6,7)$. La edad por sí misma no es una causa de disfagia, pero el aumento de la prevalencia de dichas enfermedades convierte al paciente anciano en un grupo muy vulnerable para padecer disfagia2. En el presente estudio la edad media de los pacientes fue de $83,21 \%$ años (DE 11,4 ). Según los resultados hallados la causa principal de disfagia fueron las enfermedades neurológicas $(77,23 \%)$ de las cuales un $48,72 \%$ fueron de origen vascular, destacando la demencia vascular por encima de los ACV, en contra de los resultados obtenidos en otros estudios $(2,8)$.

Otra variable analizada fue el sexo. El sexo masculino ha sido descrito por algunos autores como un factor predictor de neumonía aspirativa (9), la causa más probable de fallecimiento en los pacientes con disfagia (10), y de fallecimiento por cualquier causa en ancianos críticos (11), otros no han encontrado asociación entre la mortalidad en pacientes con disfagia y el sexo (8); sin embargo, y a diferencia de lo publicado en la literatura revisada, la mortalidad encontrada en la muestra analizada fue significativamente mayor en las mujeres $(42,3 \%)$ que en los hombres $(7,8 \%)$, manteniendo dicha diferencia tras ajustar el resultado por la edad; sin que dispongamos de una explicación plausible de este hallazgo.

Las complicaciones de la disfagia son graves y suponen una importante morbimortalidad para los pacientes que la padecen. Estas complicaciones pueden ser debidas a alteraciones de la eficacia o de la seguridad de la deglución. La alteración en la eficacia de la deglución puede conllevar a la desnutrición y deshidratación del paciente. En nuestro estudio, sólo consta que se realizara una valoración nutricional en 49 de los casos, de los cuales 28 fueron diagnosticados de desnutrición mixta, 10 pacientes de desnutrición proteica y 3 pacientes de desnutrición calórica. En el resto de los pacientes, no estaba recogido este dato; aunque en un gran porcentaje de pacientes se realizó algún tipo de intervención nutricional, de lo que se deduce que probablemente sí existe una clara concienciación sobre la necesidad de realizar este tipo de valoración, y de hecho se hace, pero no el hábito de reflejarlo en la documentación clínica; quizás por la ausencia, en nuestro centro, de un programa universal de detección de malnutrición para todos los pacientes hospitalizados impulsado y publicitado desde la dirección.

La alteración de la seguridad de la deglución implica un alto riesgo de complicaciones respiratorias secundarias a la aspiración, provocando neumonía aspirativa hasta en un $50 \%$ de los casos, con una alta tasa de mortalidad $(23-60 \%)(5,12-14)$ tasa que concuerda con los datos obtenidos en este estudio. La neumonía aspirativa, constituye la principal causa de muerte en los pacientes con enfermedades neurológicas y pacientes frágiles con alteración de la deglución (15).

Respecto al análisis por Unidades de Hospitalización destacan varios hallazgos: el primero, que la gran mayoría de ingresos por disfagia o sus complicaciones se realizaron en Geriatría lo que podría explicarse porque la disfagia se asocia al envejecimiento (16) y la edad de los pacientes ingresados en geriatría es la más 
elevada; pero tampoco puede descartarse que los geriatras estén más sensibilizados con este problema, que es considerado un síndrome geriátrico, y lo reflejen en sus informes clínicos con más frecuencia que otros profesionales (17). El mayor número porcentual de reingresos por número de hospitalizaciones en esta unidad podría estar justificado porque los pacientes ingresados en geriatría probablemente sean más fágiles18. La mayor mortalidad porcentual detectada en los hospitalizados en la Unidad de Medicina interna podría deberse a que fundamentalmente se reflejen los diagnósticos de disfagia en los pacientes más graves, ya que a pesar de que el volumen total de pacientes ingresados anualmente por cualquier causa en esta unidad es mayor que el que tiene lugar en Geriatría el número de ingresos por disfagia es claramente menor; eso en un porcentaje de casos podría ser explicado por la menor edad de sus pacientes, como se ha comentado previamente, pero no en su totalidad.

Consideramos que las fortalezas de este trabajo derivan de dos características esenciales de la muestra: su considerable tamaño, en primer lugar y, además, porque al haber incluido en ésta al total de la población hospitalizada con disfagia se elimina el sesgo de selección, importante en los estudios transversales (de prevalencia). Su principal debilidad viene condicionada justamente por el tipo de estudio, que no permite establecer relaciones de causalidad; únicamente asociaciones.

El abordaje de la disfagia debe ser multidisciplinar y protocolizado para disminuir las complicaciones $y$, por tanto, la morbimortalidad. El diagnóstico y el tratamiento precoz de la disfagia orofaríngea supondría una importante reducción de dicha morbimortalidad, mejorando la evolución de los pacientes; a corto plazo disminuyendo el número de infecciones respiratorias, y a medio y largo plazo mejorando el estado nutricional (15) y la calidad de vida de los pacientes (19); incluso con menor coste hospitalario (20).

\section{REFERENCIAS}

1. Álvarez Hernández J, Rodriguez Paradinas M, Atienza Sánchez E, Valderde. La disfagia orofaríngea en el siglo XXI. En: Álvarez Hernández J (ed). Disfagia orofaríngea: soluciones multidisciplinares. Toledo: Editorial Aula Médica, 2018.

2. Barroso J. Disfagia orofaríngea y broncoaspiración. Rev Esp Geriatr Gerontol 2009;44(S2):22-28. https://www.doi.org/10.1016/i.regg.2009.06.010

3. Camarero González E. Consecuencias y tratamiento de la disfagia. Nutr Hosp Supl. 2009;2(2):66-78

4. Alonso Martínez JL, Llorente Díez B, Echegaray Agara M, Urbieta Echezarreta MA, González Arencibia C. Reingreso hospitalario en Medicina Interna. An Med Interna (Madrid).
2001;18(5):248-254.

5. Clavé $P$, Verdaguer $A$, Arreola V. Disfagia orofaríngea en el anciano. Med Clin (Barc). 2005; 124:742-748. https://doi.org/10.1157/13075447

6. Young Gon Son, Jungho Shin, Ho Geol Ryu. Pneumonitis and pneumonia after aspiration. J Dent Anesth Pain Med 2017;17(1):1-12.

https://doi.org/10.17245/jdapm.2017.17.1.1

7. Ebihara S, Sekiya H, Miyagi M, Ebihara T, Okazaki T. Dysphagia, dystussia, and aspiration pneumonia in elderly people. J Thorac Dis. 2016;8(3):632-639. https://doi.org/10.21037/jtd.2016.02.60

8. Ferrero López MI, De la Rubia Ortí JE, Castellano Vela E, González Monte C, Sanchis-Bayarri Bernal V, Navarro Sanz R. Los factores relacionados con la mortalidad en pacientes con disfagia ayudan en la toma de decisiones dietéticas y nutricionales. Nutr Hosp. 2015;31(2): 820-828. https://doi.org/10.3305/nh.2015.31.2.7766

9. Manabe T, Teramoto S, Tamiya N, Okochi J, Hizawa N. Risk Factors for aspiration pneumonia in older adults. PLOS ONE 2015; 10(10):e0140060. https://doi.org/10.1371/journal.pone.0140060

10. Lo WL, Leu HB, Yang MCh, Wang DH, Hsu ML. Dysphagia and risk of aspiration pneumonia. A nonrandomized, pairmatched cohort study. Journal of Dental Sciences. 2019;14:241-247.

https://doi.org/10.1016/j.jds.2019.01.005

11. Giannasi SE, Venuti MS, Midley AD, Roux N, KecskesC, San Román E. Fractores de riesgo de mortalidad de los pacientes ancianos en cuidados intensivos sin limitación del esfuerzo de tratamiento. Med Intensiva. 2018; 42(8):48289. https://doi.org/10.1016/j.medin.2017.10.014

12. Lanspa MJ, Peyrani P, Wiemken T, Wilson E, Ramírez JA, Dean NC. Characteristics associated with clinician diagnosis of aspiration pneumonia; a descriptive study of afflicted patients and their outcomes. J Hosp Med. 2015; 10(2): 9096. https://doi.org/10.1002/jhm.2280

13. Ozer MN, Uzunlulu M, Oguz A, Kostek O. The effect of sociodemographic and clinical features on mortality in patients with diagnosis of aspiration pneumonia. North Clin Istanb. 2015; 2(1):41-47. https://doi.org/10.14744/nci.2015.41713

14. Jones J. Risk and outcome of aspiration pneumonia in a city hospital. J Natl Med Assoc. 1993; 85: 533-536.

15. Almiral J, Cabré M, Clavé P. Neumonía aspirativa. Med Clin (Barc). 2007; 129:424-432. https://doi.org/10.1157/13110467

16. Martin A, Ortega O, Clavé P. Disfagia orofaríngea, un nuevo síndrome geriátrico. Rev Esp Geriatr Gerontol 2018; 53:3-5.

17. Baijens LW, Clavé $P$, Cras $P$, Ekberg $O$, Forster A, Kolb GF, et al. European Societyfor Swallowing Disorders-European Union Geritatric Medicine Society whitepaper: Oropharyngeal dysphagia as a geriatric syndrome. Clin Interv Aging.2016;11: 1403-28.5.

18. Abizanda P. Actualización en fragilidad. Rev Esp Geriatr Gerontol. 2010; 45:106-110

19. Robbins JA, Langmore S, Hind JA, Erlichman M. Dysphagia research in the 21 century and beyond: proceedings form dysphagia expeerts meeting, Augist 21. Rehabil Res Dev 2002; 39:543-548

20. Matía Martín P, Cuesta Triana F. Nutrición en el anciano hospitalizado. Rev Esp Geriatr Gerontol 2006; 41:340-356 